\title{
Calcium Channel TRPV6 is a Novel Regulator in RANKL-induced Osteoclastic Differentiation and Activity through the IGF-PI3K-AKT Pathway
}

\section{Jun Ma}

Changzheng Hospital https://orcid.org/0000-0002-1796-8283

\section{Lei Zhu}

Changzheng Hospital

\section{Zhibin Zhou}

Changzheng Hospital

\section{Tengfei Song}

Changzheng Hospital

\section{Lei Yang}

Wenzhou Medical University

Xu Yan

Changzheng Hospital

\section{Aimin Chen}

Changzheng Hospital

TianWen Ye ( $\nabla$ yetianwenvip@126.com )

Department of Orthopedic Trauma Surgery, Changzheng Hospital, The Second Military Medical University, 415 Fengyang Rd, Huangpu District, Shanghai, China

\section{Research}

Keywords: osteoporosis, TRPV6, osteoclast, IGF-PI3K-AKT

Posted Date: June 3rd, 2020

DOl: https://doi.org/10.21203/rs.3.rs-29956/v1

License: (c) (i) This work is licensed under a Creative Commons Attribution 4.0 International License. Read Full License 


\section{Abstract}

Background: Calcium ion signals are important for osteoclast differentiation. Transient receptor potential vanilloid 6 (TRPV6) is a novel regulator of bone homeostasis. However, it is unclear whether TRPV6 was involved in the process of osteoclast formation.

Results: In the present study, we found that knockout of the TRPV6 gene induced osteoporosis in mice, and depletion of TRPV6 did not affect bone formation but significantly enhanced bone resorption. Further studies showed that TRPV6, distributed on the cell membrane of osteoclasts, was a negative regulator of osteoclast differentiation and function. Mechanistically, TRPV6 suppressed osteoclastogenesis by decreasing the production the IGF1R and AKT ligands to inhibit the IGF-PI3K-AKT signaling pathway. Blocking the IGF-PI3K-AKT pathway significantly reduced the effect of TRPV6 on osteoclasts.

Conclusion: Our study confirmed the important role of TPRV6 in bone metabolism and clarified its regulatory role on osteoclasts at the cellular level, which may provide a new strategy for the treatment of osteoporosis.

\section{Background}

Osteoporosis is a very common global metabolic bone disease characterized by decreased bone mineral density and bone mass ${ }^{[1,2]}$. The decreasing of bone mass is related to the abnormal differentiation and proliferation of osteoclasts and the function of bone resorption, so osteoclasts are considered to be a target cell for treating osteoporosis ${ }^{[3-5]}$. Despite the regulation of osteoclast formation and function by numerous cytokines and hormones, the macrophage colony-stimulating factor (M-CSF) and receptor activator of NF-KB ligand (RANKL) are the most critical molecules for osteoclastogenesis ${ }^{[6-9]}$. Calcium ion $\left(\mathrm{Ca}^{2+}\right)$ channels are also essential for osteoclast differentiation ${ }^{[10]}$. Extensive studies have indicated that RANKL induced oscillatory changes in intracellular $\mathrm{Ca}^{2+}$ concentrations and activated nuclear factor of activated T cells $\mathrm{c} 1$ (NFATc1), resulting in osteoclast-specific gene transcription to induce osteoclast differentiation ${ }^{[11,12]}$. However, the exact calcium signals involved in RANKL-induced osteoclast differentiation need further clarification.

Transient receptor potential vanilloid (TRPV) is a family of calcium transport proteins. Of which only TRPV5/ 6 are highly selective calcium channel proteins ${ }^{[13-15]}$. Recent studies have found that TRPV6 is an important calcium channel involved in the regulation of bone metabolism ${ }^{[16-19]}$. However, the specific molecular events remain elusive and need to be further explored.

The IGF signaling pathway widely exists in bone tissue and is involved in the regulation of bone metabolism ${ }^{[20,21]}$. Many studies have indicated that IGF1R and IGF1 proteins are expressed in osteoclasts, and IGF1R is involved in osteoclast differentiation ${ }^{[22-24]}$. However, whether the IGF signaling pathway is involved in the regulation of osteoclast formation and bone resorption function by TRPV6 remains unclear. 
In this study, we observed the potential effect and mechanism of TRPV6 in bone metabolism, osteoclast formation and bone resorption activity. In in vivo studies, we found that the depletion of TRPV6 results in severe osteoporosis in TRPV6 gene knockout mice. Inactivation of TRPV6 did not affect bone formation but significantly enhanced bone resorption. The in vitro studies revealed that TRPV6 was an important negative regulator for osteoclast differentiation and bone resorption by inhibiting the IGFIR-PI3K-AKT pathway.

\section{Materials And Methods}

\section{In vivo treatment}

Eight-week-old TRPV6 gene knockout mice and wild-type mice (from the Laboratory Animal Center of the Second Military Medical University) were used in this study. All of the mice were housed in rearing cages (five mice per cage) and kept under standard laboratory conditions (12-hour light-dark cycle; $25^{\circ} \mathrm{C}$ ). Experiments were approved by Medical Ethics Committee of the Second Military Medical University.

\section{Skeletal phenotyping}

To assess bone density and trabecular micro architecture. The distal femurs were taken for scanning using micro-computed tomographic imaging (SkyScan, Aartselaar, Belgium). The scanning analysis area was the trabecular portion of the proximal femoral growth plate from $2 \mathrm{~mm}$ downward. Microarchitectural parameters include bone mineral density (BMD), trabecular number (Tb.N) and Bone Volume/Total Volume (BV/TV).

\section{Histological analysis}

The harvested femurs were fixed in $4 \%$ paraformaldehyde solution, and decalcified in EDTA buffer saline solution. Sagittal tissue sections (10 $\mu \mathrm{m}$ thick) were prepared for immunohistochemical. HE staining, Masson trichromic staining and TRAP staining were performed afterward to assess the histological changes.

\section{Dynamic bone formation}

The femurs from mice were dehydrated with different concentrations of alcohol (70\% to $100 \%)$, and embedded in methyl-methacrylate (MMA; Sigma-Aldrich, St. Louis, MO, USA). The bone tissue was then cut and polished to a thickness of about $10 \mathrm{~mm}$. The sections were imaged using a fluorescent microscope (Carl Zeiss, Oberkochen, Germany). The mineral apposition rate (MAR) was calculated using histomorphometric data.

\section{Measurements of plasma ALP and CTX-1}

Plasma ALP and CTX-1 concentrations were determined using ELISA kits (mlbio, Shanghai, China). Samples were incubated on microporous plates for 2 hours. Next, the same volume of primary antibody 
against ALP or CTX-1 was added to each sample and incubated for another hour. This was followed by a half-hour incubation with secondary antibodies. Finally, a fluorescence microplate reader was used detect OD values $(450 \mathrm{~nm})$.

\section{Cell culture}

Bone marrow cells were isolated from the femur and tibiae of C57BL/6 or TRPV6 gene knockout C57BL/6 mice. Cells were cultured in a-MEM medium supplemented with M-CSF for 1 day. The unadherent cells were harvested and cultured in a medium containing M-CSF (50 ng/mL) for 3 days. Then the adherent cells, which were preosteoclasts, were collected. Next, a-MEM medium containing M-CSF (30 ng/mL) and RANKL (50 ng/mL) was added and allowed to incubate for 7 days to generate mature osteoclasts.

\section{Tartrate-resistant acid phosphatase staining}

TRAP staining is the most common staining method to mark mature osteoclasts. A leukocyte acid phosphatase kit was used to fix and stain the cells according to the manufacturer's instructions. Mature osteoclasts (with more than three TRAP-positive nuclei) appeared dark red and were counted by an optical microscope.

\section{Pit formation assay}

Pit formation assay was used to assess bone resorption activity. Bovine cortical bone was polished into thin slices (200 $\mu \mathrm{m}$ thick). Cell slices were then cultured on 24-well plates and induced by M-CSF and RANKL. Seven days later, the sections were placed in sterile water and washed using ultrasound for 10 min to remove the cells. This was followed by staining with toluidine blue for $5 \mathrm{~min}$. The percentage of pit areas was quantified by the ImageJ software.

\section{Cell immunofluorescence}

BMMs were induced by M-CSF and RANKL for 7 days. Then The culture medium was washed with PBS and the cells were fixed with 4\% paraformaldehyde for $20 \mathrm{~min}$. Afterwards, 0.2\% triton-x100 was applied for 5 min to induce cell permeability. After washing the permeable solution with PBS, the permeable solution was blocked with 5\% BSA for half an hour. Additionally, the primary antibodies (TRPV6, IGFIR or p-AKT) were added to incubate the cells overnight at $4^{\circ} \mathrm{C}$. Then, the PBS washed off the primary and upper corresponding secondary antibodies for $30 \mathrm{~min}$ at $37^{\circ} \mathrm{C}$. After the second antibody was washed, the cells were finally fixed with an anti-fading fixing medium. Fluorescence was observed by confocal laser scanning microscope (Leica, Germany).

\section{Lentiviral transduction and oligonucleotide transfection}

Osteoclast precursors were seeded into the 6-well plates with a density of $2 \times 10^{5}$ and transfection began when the cell fusion rate reached approximately $60 \%$. Cells were infected with TRPV6 shRNA lentiviral 
particles or TRPV6 lentiviral activation particles (Santa Cruz, CA, USA) separately for 24 hours. The culture medium was then replaced with a normal osteoclast induction medium.

\section{Quantification of mRNA and qPCR}

Total RNA was isolated from cells using TRIzol reagent (Takara Biotechnology, Japan), and cDNA was synthesized from RNA with the miScript Reverse Transcription Kit (Takara, Tokyo, Japan) according the manufacturer's protocols. qRT-PCR was carried out with the SYBR Green PCR kit (Takara Biotechnology, Japan) and sequence detection was performed using an ABI 7500 Sequencing Detection System (Applied Biosystems, Foster City, CA). $\beta$-actin was used as the housekeeping gene. The primers used are described in Table 1.

\section{Western blot analysis}

Proteins the from osteoclast lysates were introduced to $10 \%$ SDS-PAGE, and were subsequently electroblotted onto a polyvinylidene fluoride membrane. The membrane was blocked with $5 \%$ fat-free milk and incubated with appropriate antibodies. Chemiluminescence reagents (Thermo Scientific, Rockford, IL) were used to detected antigen-antibody complexes. ImageJ software was used to quantify the immunoblots.

\section{Statistical analysis}

Data are shown as mean \pm SD. SPSS24.0 statistical software was used for statistical analysis. The two tailed non-paired Student's $t$ test and one-way ANOVA were used to compare differences. Statistical significance was set at $P<0.05$ and very significance was $P<0.01$.

\section{Results}

\section{Knockout of the TRPV6 gene induces osteoporosis in mice}

TRPV6 gene knockout mice were used in this study to investigate the biological role of TRPV6 in bone metabolism. To confirm whether the TRPV6 depletion mouse model was successfully constructed, we compared the transcription levels of mRNA TRPV6 in bone marrow monocytes from wild-type mice and TRPV6 $^{-/-}$mice. The result showed that the expression of TRPV6 was significantly depleted in TRPV6 ${ }^{-/-}$ mice. Furthermore, there were no significant differences in the expression of mRNA TRPV2, mRNA TRPV4 and mRNA TRPV5 in bone marrow monocytes from TRPV6 ${ }^{-/-}$mice and WT mice (Fig. 1A). At 12 weeks old, mice were euthanized and femur cancellous bone was analyzed by $\mu \mathrm{CT}$. The results revealed that TRPV6 $^{-/-}$mice had remarkably decreased BMD (Fig. 1B). Representative $\mu \mathrm{CT}$ images of trabecular bone are presented in Fig. 1C. Analysis of the trabecular structure revealed that the Tb.N and BV/TV were significantly lower in TRPV6 ${ }^{-/-}$mice (Fig. 1D-E), which is consistent with the results of HE staining (Fig. $1 F)$. 
Abnormal bone formation or resorption leads osteoporosis. At the beginning of this study of the effect of TRPV6 deletion on osteogenic function, the number of osteoblasts (N.Ob/BS) in TRPV6 ${ }^{-/-}$mice was similar to that in WT mice, which was calculated using Masson trichromic staining (Fig. 2A, B). Similar results were attained for the tetracycline double-standard staining, and the mineral apposition rate (MAR) was comparable between the two groups (Fig. 2C, D). In addition, there was no significant difference in serum ALP concentration between TRPV6 ${ }^{-/-}$mice and WT mice (Fig. 2E). TRAP staining in the bone sections further revealed the effect of TRPV6 depletion on osteoclastogenesis (Fig. 2F). Quantification showed that the number and surface area of osteoclasts in TRPV6 ${ }^{-/-}$mice increased significantly (Fig. 2G). Compared with WT mice, TRPV6 ${ }^{-/-}$mice had higher levels of CTX-1. To summarize, these results suggest that bone absorption was enhanced and bone formation remained unchanged in $\mathrm{TRPV}^{-/-}$mice.

\section{TRPV6 negatively regulates osteoclast differentiation and fusion, inhibiting osteoclast formation}

The results of western blotting suggest that TRPV6 is expressed in osteoclasts. The expression of TRPV6 in osteoclasts was reduced during cell differentiation (Fig. 3A). In addition, immunocytochemical fluorescence indicated that TRPV6 mainly distributes on the membrane of mature osteoclast (Fig. 3B). For deeper insight into the role of TRPV6 on osteoclast function, TRAP staining was first applied, in cultures derived from TRPV6 ${ }^{-/-}$mice, and the number of TRAP ${ }^{+}$multinucleated osteoclasts were significantly higher (Fig. 3C-D). A bone resorption lacuna experiment result showed that resorption pits were significantly enhanced in TRPV6 ${ }^{-/-}$osteoclasts (Fig. 3E-F). In addition, PCR results revealed that the mRNA levels of marker genes for osteoclastogenesis (cathepsin k, DC-STAMP, Atp6v0d2 and TRAP) were obviously upregulated in TRPV6-depleted osteoclasts (Fig. 3G-J).

\section{Silencing of TRPV6 enhanced osteoclast formation}

To further verify the role of TRPV6 on osteoclast formation and bone resorption, we silenced and overexpressed TRPV6 gene in osteoclasts by lentivirus transfection. Almost all cells expressed GFP, indicating that the lentivirus transfection rate was more than 95\% (Fig. 4A). Western blotting confirmed that TRPV6 was effectively silenced and overexpressed (Fig. 4B). The mRNA levels of cathepsin k, DCSTAMP, Atp6v0d2 and TRAP were all increased in osteoclasts infected with lenti-shRNA-TRPV6, whereas were all decreased in the group of osteoclast infected with lenti-pMX-TRPV6 (Fig. 4C-F). Next, we assessed the differentiation of osteoclasts with silenced or overexpressed TRPV6 by TRAP staining assay. The number of stained multinuclear TRAP ${ }^{+}$osteoclasts was obviously increased in osteoclasts with silenced TRPV6 in a time-dependent manner, whereas osteoclasts showed decreases in the TRPV6overexpressed group (Fig. 4G-H). The above results strongly suggest that TRPV6 was a negative regulator of osteoclast differentiation and function.

\section{The IGF pathway is involved in the negative regulation of osteoclast formation and resorption by TRPV6}

Our previous study revealed that the RANKL-induced $\left[\mathrm{Ca}^{2+}\right.$ ]i oscillation response was not significantly affected by inhibition of TRPV6 ${ }^{[16]}$. As a non-ca ${ }^{2+}$ oscillating signaling pathway, the IGF pathway plays 
an important role in osteoclast formation ${ }^{[25-27]}$. Therefore, we speculated that the IGF signaling pathway was potentially involved in the regulation of osteoclast differentiation and bone absorption by TRPV6. The results showed that the levels of IGF1R and IGFBP1 mRNA and protein in osteoclasts were significantly increased after TRPV6 gene silencing (Fig. 5A-D), which confirmed our speculation. Next, we used an IGF1R antagonist NVP-AEW541 to block the IGF signaling pathway, TRAP staining demonstrated that the inhibitory effect of TRPV6 on osteoclast differentiation was weakened in osteoclasts with blockers (Fig. 5E-F). In accordance with the results for TRAP staining, the IGF1R blocker significantly inhibited the induction of the bone resorption of osteoclasts by silencing of TRPV6, as evidenced by the pit formation assay (Fig. 5G-H). These results strongly suggest that TRPV6 negatively regulated osteoclast formation and bone resorption by inhibiting the IGF pathway.

\section{TRPV6 negatively regulates osteoclast formation and resorption by inhibiting the IGF1R-PI3K-AKT pathway}

As assessed by immunofluorescence, we found that osteoclasts highly expressed TRPV6 and IGF1R in the membrane and cytoplasm [Fig. 6A]. To further explore the downstream signaling of the IGF pathway, BMMs isolated from TRPV6 ${ }^{-/-}$and WT mice were induced by RANKL and M-CSF for 7 days to form mature osteoclasts. The levels of phosphorylated markers P85/p-P85, PDK1/p-PDK1 and AKT/p-AKT in the PI3K-AKT pathway were detected by western blotting. The results showed that the ratios of P85/pP85, PDK1/p-PDK1 and AKT/p-AKT were increased in osteoclasts isolated from TRPV6 ${ }^{-/-}$mice compared with WT mice [Fig. 6B-E]. Consistent with the western blotting results, immunofluorescence showed that the ratio of PDK1/p-PDK1 of osteoclasts isolated from TRPV6 ${ }^{-/-}$mice was higher than that of osteoclasts isolated from WT mice [Fig. 6F-G]. Next, we used NVP-AEW541 to block the IGF signaling pathway and western blotting revealed that there was no significant difference in the level of AKT/p-AKT between the osteoclasts derived from TRPV6 ${ }^{-/-}$mice and WT mice [Fig. $\left.6 \mathrm{H}-1\right]$.

\section{Discussion}

In the present study, we demonstrated that TRPV6 was a critical negative regulator in RANKL-induced osteoclast differentiation and activity. Our studies showed that TRPV6 decreased osteoclast formation and bone resorption by inhibiting the IGF-PI3K-AKT signaling pathway (Fig. 7).

Bone metabolism maintains bone mass through complex regulation of the balance between bone resorption and formation ${ }^{[28-31]}$. As a highly selective calcium channel in the TRPV subfamily, TRPV6 is an important protein in osteoclasts and participates in the regulation of osteoclast differentiation and bone resorption ${ }^{[16]}$. Bianco et al ${ }^{[32]}$ found that TRPV6 ${ }^{-/-}$mice had obvious bone metabolism disorders, manifested as intestinal calcium absorption dysfunction and decreased bone density. Lieben et al reported that the bone mass of TRPV6 ${ }^{-1-}$ mice fed a low calcium diet decreased significantly and bone resorption and bone formation were enhanced simultaneously, with bone resorption being more active than bone formation ${ }^{[33]}$. In this study, we found that BMD, Tb.N and BV/TV were obviously decreased in 
TRPV6-depleted mice, suggesting that TRPV6 is involved in the regulation of bone metabolism.

Furthermore, Masson trichromic staining showed that N.Ob/BS was not decreased in TRPV6 ${ }^{-/-}$. Similar results were revealed by tetracycline double-standard staining, MAR was comparable between the two groups; however, TRPV6 ${ }^{-/-}$mice had significantly more TRAP positive cells in the metaphyseal region of femoral bone sections than WT mice. Therefore, based on the above findings, we believed that TRPV6 contributes to bone homeostasis through the regulation of osteoclast differentiation and activity.

Previous studies have confirmed that in the presence of M-CSF and RANKL sufficient to maintain cell survival and induce complete osteoclastic differentiation ${ }^{[34,35]}$. Several reports showed that TRPV6 regulates a variety of cellular functions, including differentiation, proliferation and apoptosis, by affecting intracellular $\mathrm{Ca}^{2+}$ concentration ${ }^{[36]}$. The increased concentration of $\mathrm{Ca}^{2+}$ in osteoclasts causes the cell pseudopodia to retract, restrict its movement, destroy the absorption skeleton structure, and thus inhibit bone absorption ${ }^{[37]}$. In our study, we found that TRPV6 expression was decreased in a time-dependent manner during the process of osteoclast differentiation, and the distribution of TRPV6 in osteoclasts was identified as being mainly distributed on the cell membrane. In addition, TRAP staining and a bone absorption pit experiment showed that the differentiation and fusion of osteoclasts isolated from TRPV6 $^{-/-}$mice was significantly quicker than that from WT mice. We also found that silencing TRPV6 in osteoclasts significantly increased osteoclastogenesis. Thus, it is likely that the inhibitory role of TRPV6 in osteoclastic resorption is caused by decreasing osteoclastogenesis.

Both independent signaling pathways and calcineurin-dependent pathways contribute to NFATC1 activation, leading to efficient osteoclastogenesis ${ }^{[38,39]}$. Our previous research found that non- $\mathrm{Ca}^{2+}$ oscillation signaling pathways contribute to TRPV6 deficiency-induced osteoclastogenesis ${ }^{[16]}$. As an important non- $\mathrm{Ca}^{2+}$ oscillating pathway, the IGF signaling pathway is widely expressed in bone tissue and participates in the regulation of bone metabolism ${ }^{[40,41]}$. Dai et al.${ }^{[42]}$ found that TRPV5 negatively regulated the proliferation of NaR cells by inhibiting the IGF signaling pathway in culture medium with a normal calcium concentration. Our results showed that silencing of TRPV6 significantly increased the expression of two key proteins of the IGF signaling pathway, IGF1R and IGFBP1, in osteoclasts. Blocking IGF1R suppresses the increase of osteoclastic differentiation induced by inhibition of TRPV6. Taken together, we presume that TRPV 6 is responsible for osteoclast formation and resorption through inhibition of the IGF signaling pathway.

As a downstream signaling pathway of the cascade reaction of the IGF signaling pathway, PI3K-AKT plays an important role in the activation of osteoclasts. AKT promotes osteoclast survival by regulating cell cytoskeletal replacement and movement, and knockdown AKT reduces the expression of osteocalcin ${ }^{[43]}$. Lee et al. ${ }^{[44]}$ revealed that PI3K, p38 and extracellular signal-regulated kinase pathways were involved in osteoclast differentiation. Xing et al. reported that the PI3K-AKT signaling pathway was involved in RANKL-independent osteoclastogenesis ${ }^{[45]}$. In the present study, TRPV6 inhibited the phosphorylation level of PI3K-AKT in osteoclasts and reduced the expression of P85/p-P85, PDK1/p-PDK1 and AKT/pAKT. The effect of TRPV6 was eliminated after pretreatment with an IGF1R inhibitor (NVP-AEW541). 
In conclusion, our study confirmed the important role of TPRV6 in bone metabolism, clarified its regulatory role on osteoclasts at the cellular level, and revealed the molecular mechanism of TRPV6's negative regulation of osteoclast formation and bone absorption. These results may provide a new strategy for the treatment of osteoporosis.

\section{Declarations}

Ethics approval and consent to participate: Experiments were approved by Medical Ethics Committee of the Second Military Medical University.

Consent for publication: Not available.

Availability of data and materials: All data generated or analyzed during this study are included in this published article (and its additional files).

Competing interests: The authors declare that they have no competing interests.

Funding: This study was sponsored by the National Natural Science Foundation of China (No. 81672202 and No.81874003), the "Outstanding Young Scholar Program" and the "Doctoral innovation fund" supported by Second Military Medical University, and the "Research Clinician Program" supported by Shanghai Changzheng Hospital.

Authors' contributions: JM conception and design, collection and assembly of data, data analysis and interpretation, manuscript writing; LZ, ZBZ and TFX: collection and/ or assembly of data, data analysis; $\mathrm{LY}$ : data analysis ; $\mathrm{XY}, \mathrm{AMC}$ : conception and design, data analysis and interpretation, financial support, manuscript writing; TWY: conception and design, data analysis and interpretation, financial support, manuscript writing, final approval of manuscript. All authors read and approved the final manuscript.

Acknowledgements: Not available.

\section{Table}

Table1: Premiers for qRT-PCR analysis 


\begin{tabular}{|c|c|c|}
\hline Genes & Orientation & Sequence ( $5^{\prime}$ to $\left.3^{\prime}\right)$ \\
\hline \multirow[t]{2}{*}{ TRPV6 } & Forward & CCCAAGCTTATTTTACTGAATTCT \\
\hline & Reverse & CGGGGTACCCTAGTAGGCCCAG \\
\hline \multirow[t]{2}{*}{ TRPV5 } & Forward & ATGGGGGCTAAAACTCCTTGG \\
\hline & Reverse & ССTCTTTGCCGGAAGTCACA \\
\hline \multirow[t]{2}{*}{ TRPV2 } & Forward & AGCCATTCССTCATCAAAAG \\
\hline & Reverse & AGCCAGCTCACCCATACC \\
\hline \multirow[t]{2}{*}{ TRPV4 } & Forward & CGTCCAAACCTGCGAATGAAGTTC \\
\hline & Reverse & ССТССАTСTСTTGTTGTCACTGG \\
\hline \multirow[t]{2}{*}{ CathepsinK } & Forward & GAAGAAGACTCACCAGAAGCAG \\
\hline & Reverse & TCCAGGTTATGGGCAGAGATT \\
\hline \multirow[t]{2}{*}{ TRAP } & Forward & САСТСССАСССTGAGATTTGT \\
\hline & Reverse & CATCGTCTGCACGGTTCTG \\
\hline \multirow[t]{2}{*}{ Atp6v0d2 } & Forward & TGCGGCAGGCTCTATCCAGAGG \\
\hline & Reverse & CCACTGCCACCGACAGCGTC \\
\hline \multirow[t]{2}{*}{ DC-STAMP } & Forward & GGGGACTTATGTGTTTCCACG \\
\hline & Reverse & ACAAAGCAACAGACTCCCAAAT \\
\hline \multirow[t]{2}{*}{ IGF1R } & Forward & ACCGGGATCTCATCAGCTTCAC \\
\hline & Reverse & TCCTTGTTCGGAGGCAGGTC \\
\hline \multirow[t]{2}{*}{ IGFBP1 } & Forward & TACTATCTACTCAGAAAGTCGTGAC \\
\hline & Reverse & ACACATATATAAAATGGTGTGCTCC \\
\hline \multirow[t]{2}{*}{$\beta$-actin } & Forward & GGCTGTATTCCCCTCCATCG \\
\hline & Reverse & CCAGTTGGTAACAATGCCATGT \\
\hline
\end{tabular}

\section{References}

[1] Klein-Nulend J, et al. Bone cell mechanosensitivity, estrogen deficiency, and osteoporosis. J Biomech.2015;48(5): 855-865.

[2] Jun ma, et al. Sodium hydrosulfide mitigates dexamethasone-induced osteoblast dysfunction by interfering with mitochondrial function. Biotechnol Appl Biochem.2019; 66(4):690-697. 
[3] Hemmatian H, et al. Aging, Osteocytes, and Mechanotransduction. Curr Osteoporos Rep.2017; 15(5): 401-411.

[4] Rodan GA, Martin TJ. Therapeutic approaches to bone diseases. Science.2000; 289:1508-1514.

[5] Teitelbaum SL. Bone resorption by 0steoclasts. Science.2000; 289 :1504-1508.

[6] Ikebuchi, Y. et al. Coupling of bone resorption and formation by RANKL reverse signalling. Nature,2018; $561,195-200$.

[7] Kong YY, et al. Opgl is a key regulator of osteoclastogenesis lymphocyte development and lymph-node organogenesisi. Nature.1999; 397: 315-323.

[8] Karsenty G. Convergence between bone and energy homeostases: Leptin regulation of bone mass. Cell Metab.2006; 4: 341-348.

[9] Jeong E, et al. STAC2 negatively regulates osteoclast formation by targeting the RANK signaling complex. Cell Death Differ.2018; 25:1364-1374.

[10] Masuyama R, et al. Trpv4-mediated calcium influx regulates terminal differentiation of osteoclasts. Cell Metab.2008; 8:257-265.

[11] Hasegawa $\mathrm{H}$, et al. Serum calcium-decreasing factor, caldecrin, inhibits osteoclast differentiation by suppression of nfatc1 activity. J Biol Chem.2010; 285:25448-25457.

[12] Kim MS, et al. Rankl-mediated reactive oxygen species pathway that induces long lasting ca2+ oscillations essential for osteoclastogenesis. J Biol Chem.2010; 285:6913-6921.

[13] Weber K, et al. Gene structure and regulation of the murine epithelial calcium channels ECaC1 and 2. Biochem Biophys Res Commun.2001; 289(5): 1287-1294.

[14] Schoeber JP, Hoenderop JG, Bindels RJ. Concerted action of associated proteins in the regulation of TRPV5 and TRPV6. Biochem-Soc-Trans.2007;35(1):115-119.

[15] Tengfei Song, et al. Regulation of TRPV5 transcription and expression by E2/ERa signalling contributes to inhibition of osteoclastogenesis. J Cell Mol Med.2018; 22(10):4738-4750.

[16] Chen F, et al. Knockout of TRPV6 causes osteopenia in mice by increasing osteoclastic differentiation and activity. Cell Physiol Biochem.2014; 33(3): 796-809.

[17] Bary EL. Expression of mRNA for the alpha 1 subunit of voltage-gated calcium channels in human osteoblast-like cell lines and normal human osteoblasts. Calcif Tissue Int.2000; 66(2): 145-150. [18] Haussler MR, et al. Molecualr mechanisms of vitamin D action.2013; Calcif Tissue Int,92(2). [19] Barthel TK, et al. 1,25-Dihydroxyvitamin D3/VDR-mediated induction of FGF23 as well as transcriptional control of other bone anabolic and catabolic genes that orchestrate the regulation of phosphate and calcium mineral metabolism. J Steroid Biochem Mol Biol.2007;103(3-5): 381-388. 
[20] Bikle DD, et al. Role of IGF-I signaling in muscle bone interactions. Bone.2015; 80: 79-88.

[21] Yan J, et al.Gut microbiota induce IGF-1 and promote bone formation and growth. Proc Natl Acad Sci USA.2016;113(47).

[22] Duan C, Xu Q. Roles of insulin-like growth factor (IGF) binding proteins in regulating IGF actions. Gen Comp Endocrinol.2005;142(1-2): 44-52.

[23] Wang Y, et al. Role of IGF-I signaling in regulating osteoclastogenesis. J Bone Miner Res.2006;21(9):1350-1358.

[24] Bikle DD, Wang Y. Insulin like growth factor-l: a critical mediator of the skeletal response to parathyroid hormone. Curr Mol Pharmacol. 2012;5(2): 135-142.

[25] Xian L, et al. Matrix IGF-1 maintains bone mass by activation of mTOR in mesenchymal stem cells. Nat Med.2012; 18(7):1095-1101.

[26] Wang X, et al. A Liver-Bone Endocrine Relay by IGFBP1 Promotes Osteoclastogenesis and Mediates FGF21-Induced Bone Resorption. Cell Metab.2015;22(5): 811-24.

[27] DeMambro VE, et al. Insulin-like growth factor-binding protein-2 is required for osteoclast differentiation. J Bone Miner Res.2012;27(2): 390-400.

[28] Katsimbri P. The biology of normal bone remodelling. Eur J Can Care.2017;26:e12740.

[29] Ma J, et al. Hydrogen sulfide is a novel regulator implicated in glucocorticoids-inhibited bone formation. Aging-us.2019;11(18):7537-7552.

[30] Jun Ma, et al. Resveratrol Attenuates Lipopolysaccharides (LPS)-Induced Inhibition of Osteoblast Differentiation in MC3T3-E1 Cells. Med Sci Monit.2018;24:2045-2052.

[31] Jun Ma,et al. Metformin promotes differentiation of Human Bone Marrow Derived Mesenchymal Stem Cells into osteoblast via GSK3 $\beta$ inhibition. EUR REV MED PHARMAC0.2018;22(22): 7962-7968.

[32] Bianco SD, et al. Marked disturbance of calcium homeostasis in mice with targeted disruption of the Trpv6 calcium channel gene. J Bone Miner Res.2007;22(2): 274-285.

[33] Lieben L, et al. Trpv6 mediates intestinal calcium absorption during calcium restriction and contributes to bone homeostasis. Bone.2010; 47(2): 301-308.

[34] Boyle WJ, Simonet WS, Lacey DL. Osteoclast differentiation and activation. Nature.2003;423(6937):337-342.

[35] Robling AG, Castillo AB, Turner $\mathrm{CH}$. Biomechanical and molecular regulation of bone remodeling. Annu Rev Biomed Eng.2006;8(1):455-489. 
[36] Hoenderop JG, et al. Function and expression of the epithelial $\mathrm{Ca}(2+)$ channel family: comparison of mammalian ECaC1 and 2. J Physiol.2001;537:747-761.

[37] Lapierre DM, et al. Lysophosphatidic acid signals through multiple receptors in osteoclasts to elevate cytosolic calcium concentration, evoke retraction, and promote cell survival. J Biol Chem.2010; 285(33): 25792-25801.

[38] Chang EJ, et al. The jnk-dependent camk pathway restrains the reversion of committed cells during osteoclast differentiation. J Cell Sci.2008;121:2555-2564.

[39] van der Eerden BC, Weissgerber P, Fratzl-Zelman N. The transient receptor potential channel trpv6 is dynamically expressed in bone cells but is not crucial for bone mineralization in mice. J Cell Physiol.2012;227:1951-1959.

[40] Zhou X, et al. Three-Dimensional Printed Titanium Scaffolds Enhance Osteogenic Differentiation and New Bone Formation by Cultured Adipose Tissue-Derived Stem Cells Through the IGF1R/AKT/Mammalian Target of Rapamycin Complex 1 (mTORC1) Pathway. Med Sci Monit.2019;27;25:8043-8054.

[41] Tagliaferri C, et al. Muscle and bone, two interconnected tissues. Ageing Res Rev.2015;21:55-70.

[42] Dai W, et al. Calcium deficiency-induced and TRP channel-regulated IGF1R-PI3K-Akt signaling regulates abnormal epithelial cell proliferation. Cell Death Differ.2014;21(4): 568-81.

[43] Chen LL, et al. PI3K/AKT pathway involvement in the osteogenic effects of osteoclast culture supernatants on preosteoblast cells. Tissue Eng Part A.2013;19(19-20):2226-2632.

[44] Lee SE, et al. The phosphatidylinositol 3-kinase, p38, and extracellular signal-regulated kinase pathways are involved in osteoclast differentiation. Bone.2002;30:71-77.

[45] Xing R, et al. Interleukin-21 promotes osteoclastogenesis in RAW264.7 cells through the PI3 K/AKT signaling pathway independently of RANKL. Int J Mol Med.2016;38(4):1125-1134.

\section{Figures}


A

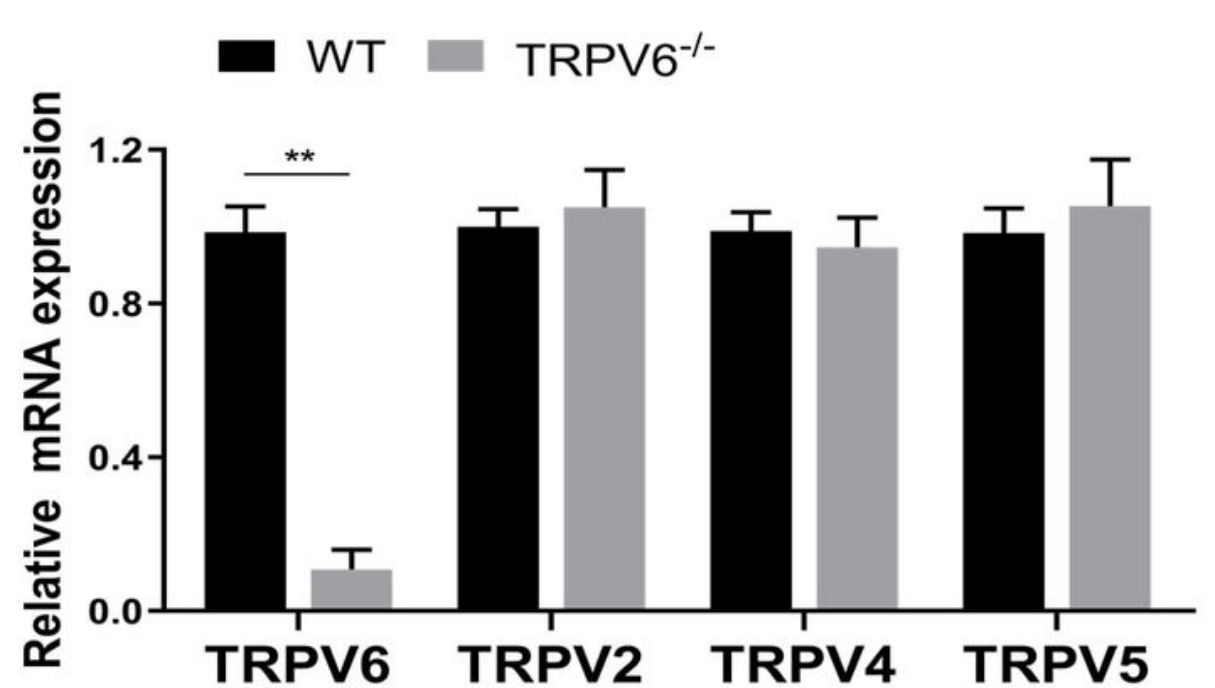

B

C

WT
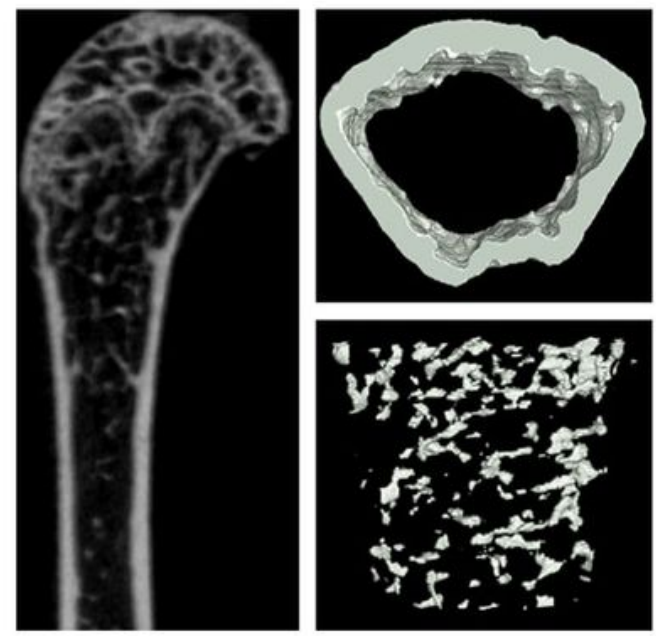

$\mathrm{F}$

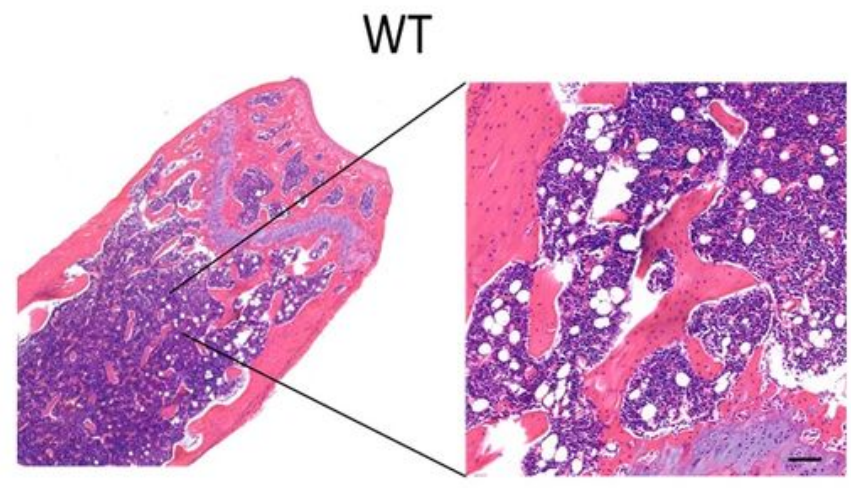

TRPV6 $\%$
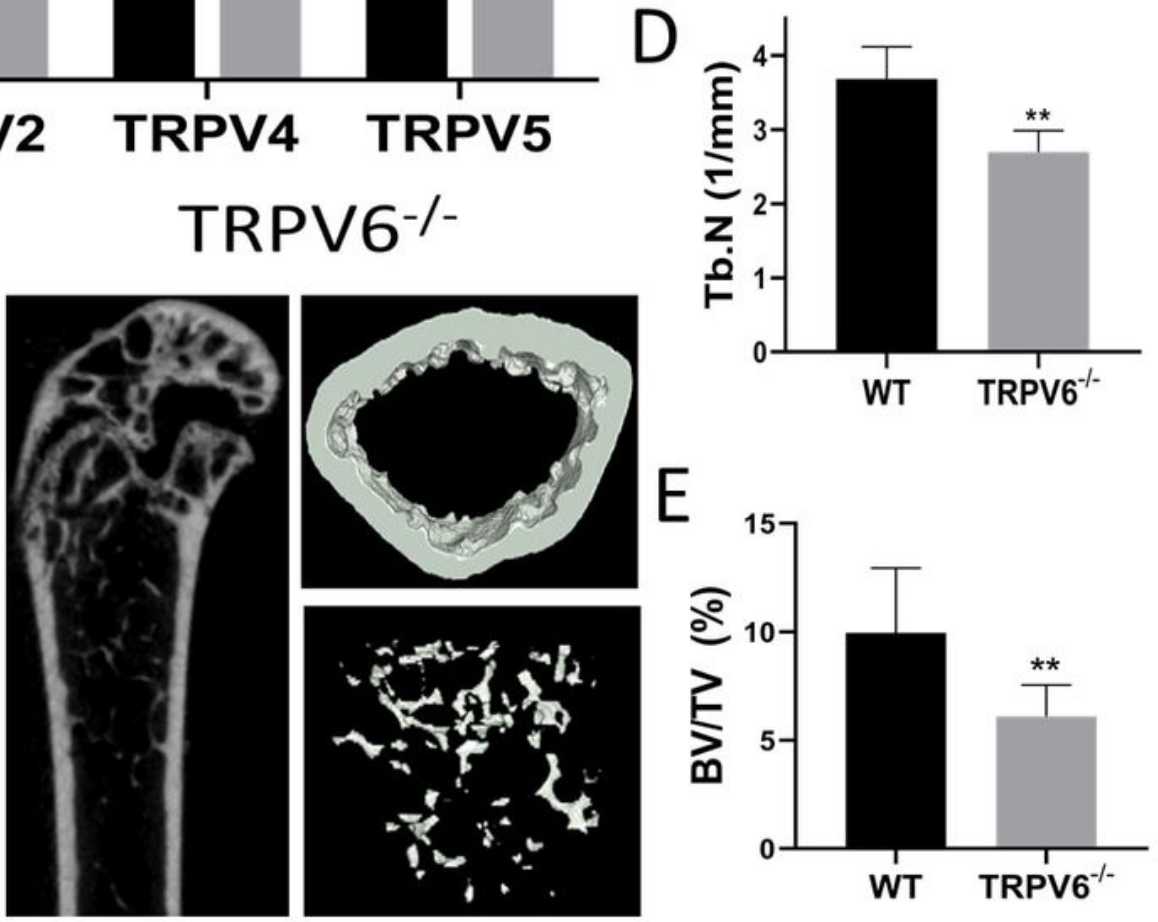

TRPV6 $\%$

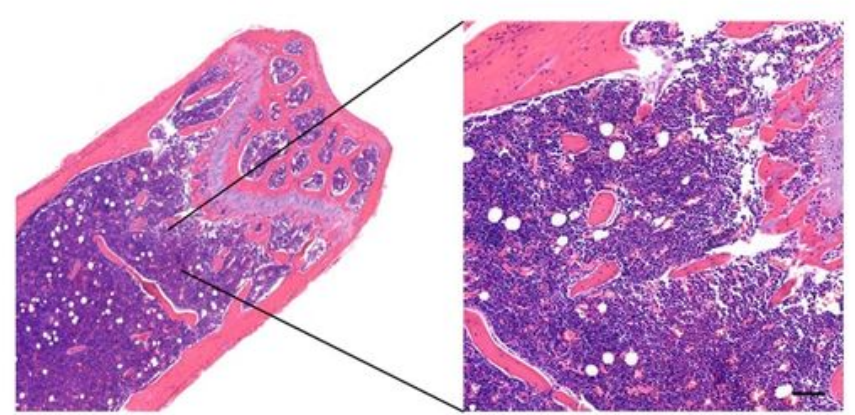

Figure 1

Furthermore, there were no significant differences in the expression of mRNA TRPV2, mRNA TRPV4 and mRNA TRPV5 in bone marrow monocytes from TRPV6-/- mice and WT mice (Fig. 1A). At 12 weeks old, mice were euthanized and femur cancellous bone was analyzed by $\mu \mathrm{CT}$. The results revealed that TRPV6-/- mice had remarkably decreased BMD (Fig. 1B). Representative $\mu$ CT images of trabecular bone are presented in Fig. 1C. Analysis of the trabecular structure revealed that the Tb.N and BV/TV were 
significantly lower in TRPV6-/- mice (Fig. 1D-E), which is consistent with the results of HE staining (Fig. 1F).
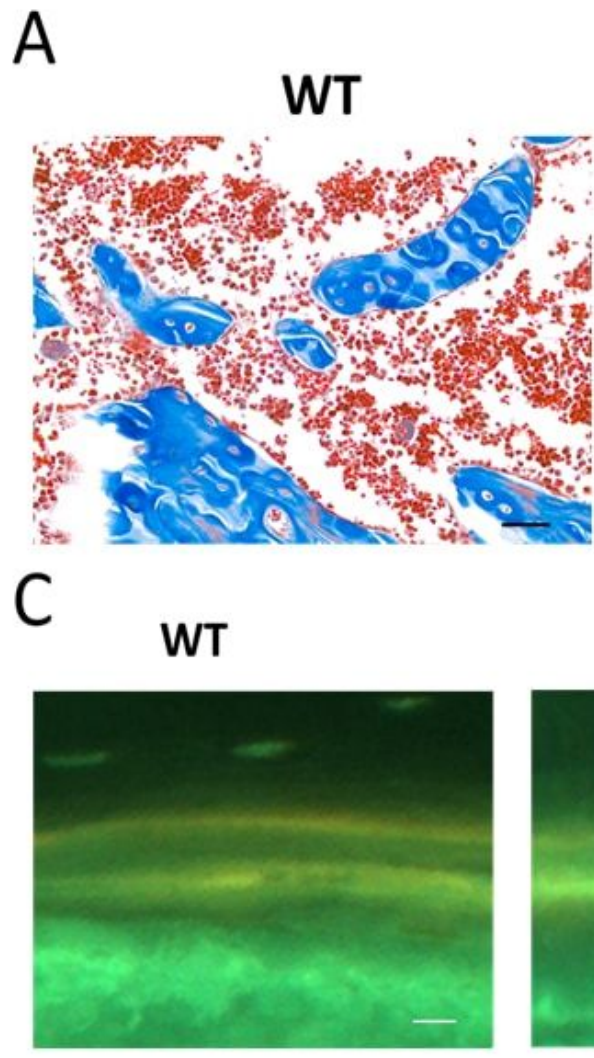

$\mathrm{F}$

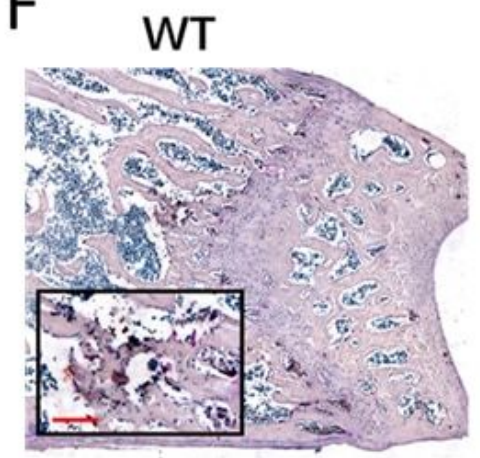

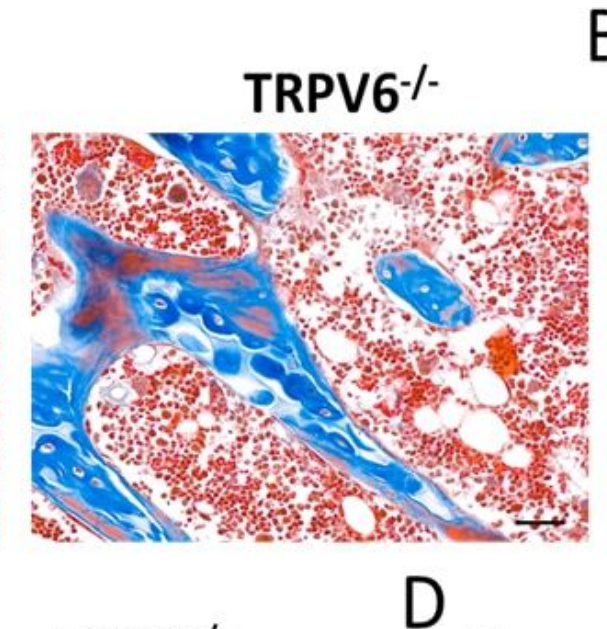

TRPV6 $\%$
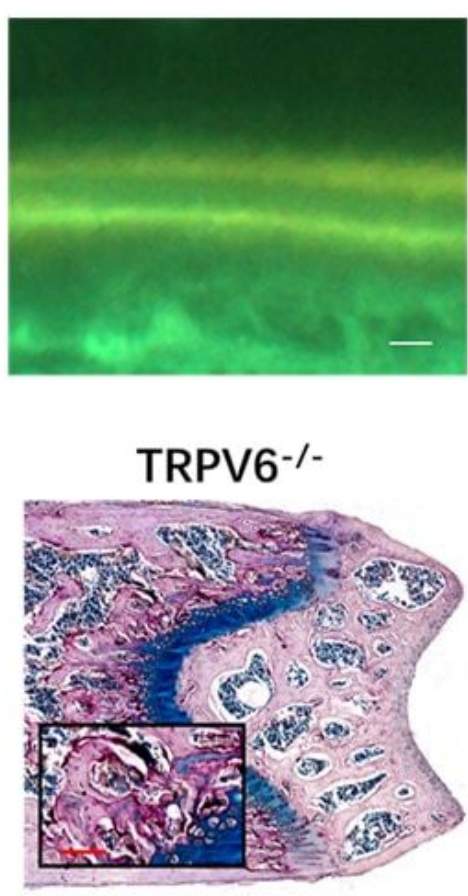

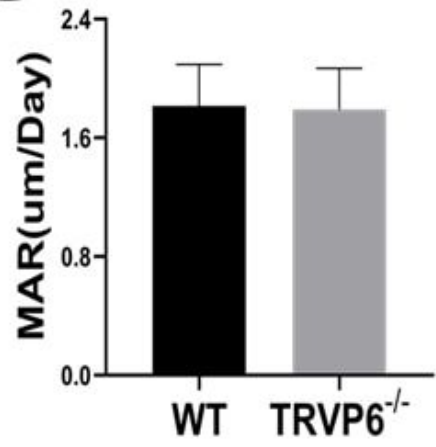

G

B
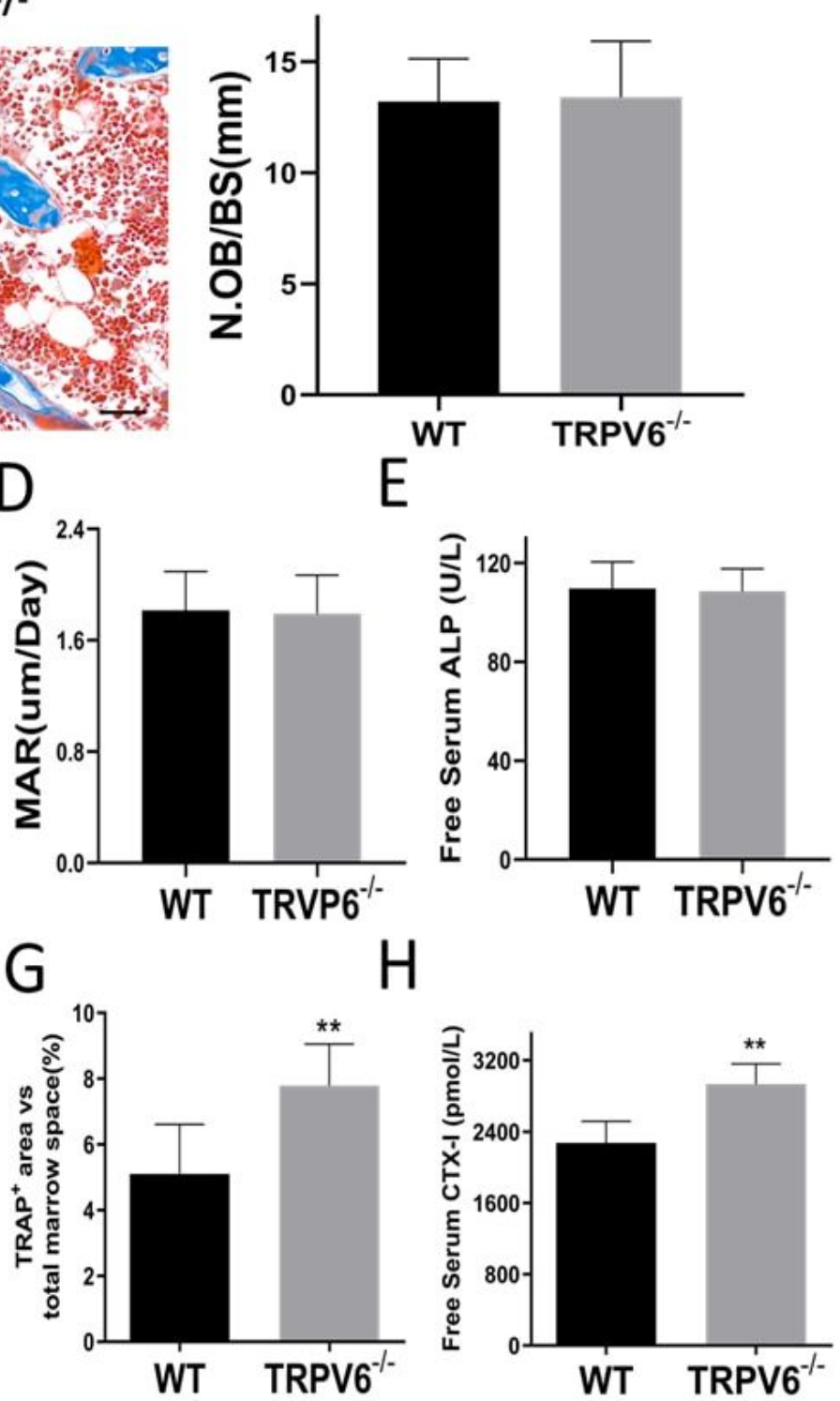

Figure 2

Abnormal bone formation or resorption leads osteoporosis. At the beginning of this study of the effect of TRPV6 deletion on osteogenic function, the number of osteoblasts (N.Ob/BS) in TRPV6-/- mice was similar to that in WT mice, which was calculated using Masson trichromic staining (Fig. 2A, B). Similar results were attained for the tetracycline double-standard staining, and the mineral apposition rate (MAR) was comparable between the two groups (Fig. 2C, D). In addition, there was no significant difference in serum ALP concentration between TRPV6-/- mice and WT mice (Fig. 2E). TRAP staining in the bone sections further revealed the effect of TRPV6 depletion on osteoclastogenesis (Fig. 2F). Quantification showed that the number and surface area of osteoclasts in TRPV6-/- mice increased significantly (Fig. $2 \mathrm{G}$ ). Compared with WT mice, TRPV6-/- mice had higher levels of CTX-1. To summarize, these results 
suggest that bone absorption was enhanced and bone formation remained unchanged in TRPV6-/mice.

A
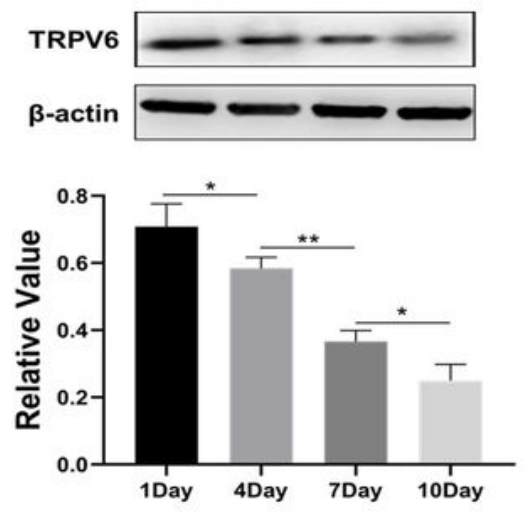

C

4 Day
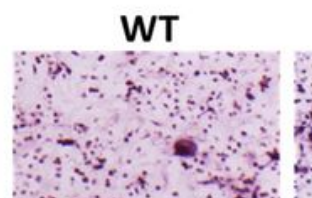

4345
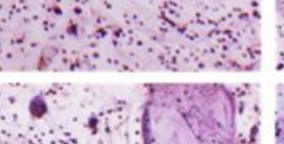

7 Day

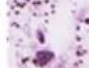

10 Day

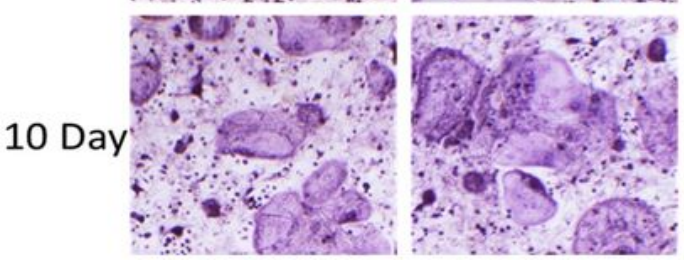

z 2 क

$342=2 \%$

D

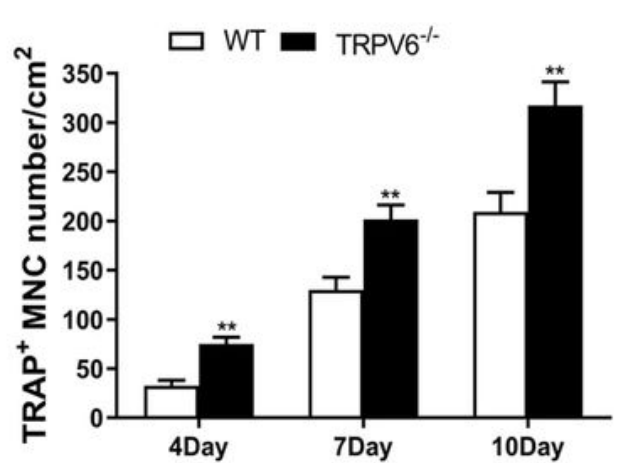

B
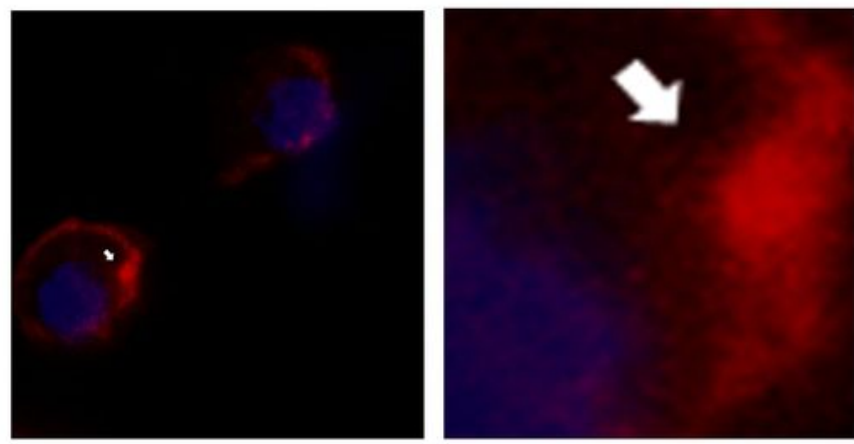

$\mathrm{E}$

4 Day

Day

10

Day

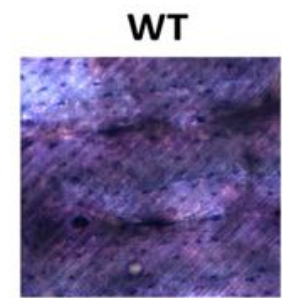

TRPV6 $\%$
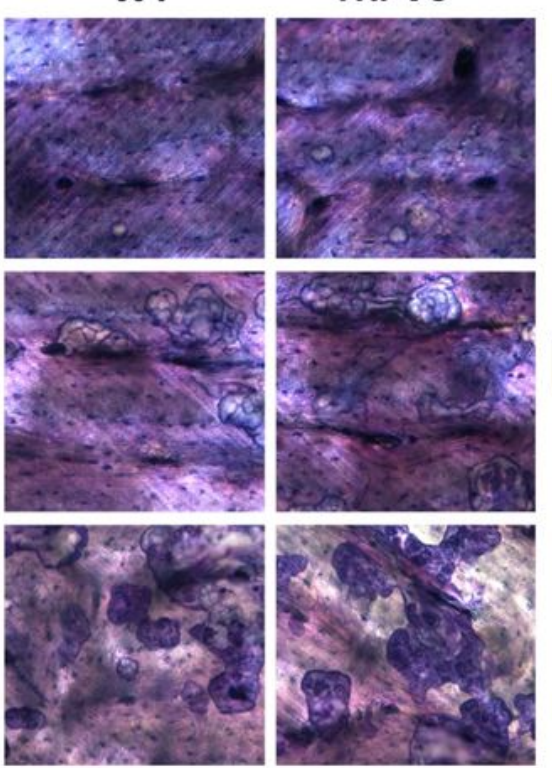

$\mathrm{F}$

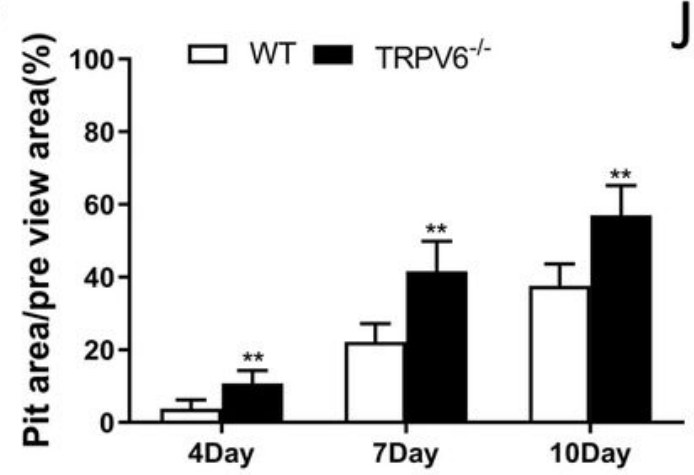

G

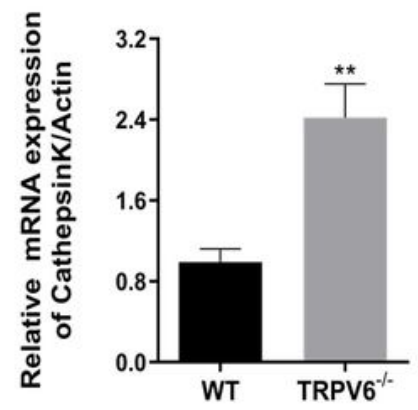

$\mathrm{H}$
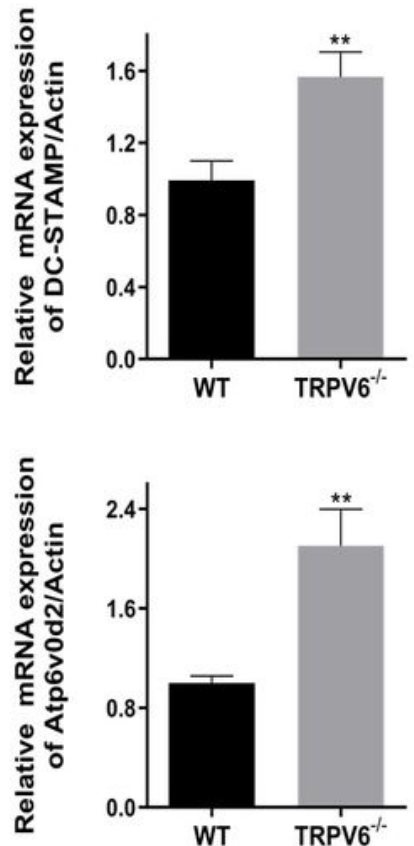

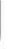

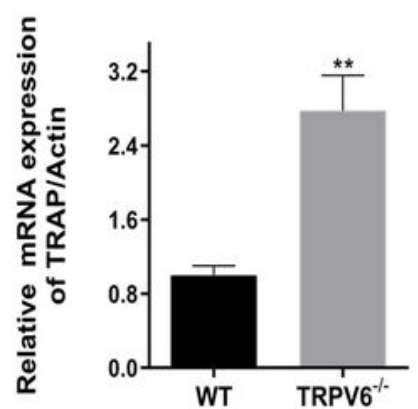

\section{Figure 3}

The results of western blotting suggest that TRPV6 is expressed in osteoclasts. The expression of TRPV6 in osteoclasts was reduced during cell differentiation (Fig. 3A). In addition, immunocytochemical fluorescence indicated that TRPV6 mainly distributes on the membrane of mature osteoclast (Fig. 3B). 
For deeper insight into the role of TRPV6 on osteoclast function, TRAP staining was first applied, in cultures derived from TRPV6-/- mice, and the number of TRAP+ multinucleated osteoclasts were significantly higher (Fig. 3C-D). A bone resorption lacuna experiment result showed that resorption pits were significantly enhanced in TRPV6-/- osteoclasts (Fig. 3E-F). In addition, PCR results revealed that the mRNA levels of marker genes for osteoclastogenesis (cathepsin k, DC-STAMP, Atp6v0d2 and TRAP) were obviously upregulated in TRPV6-depleted osteoclasts (Fig. 3G-J).

A
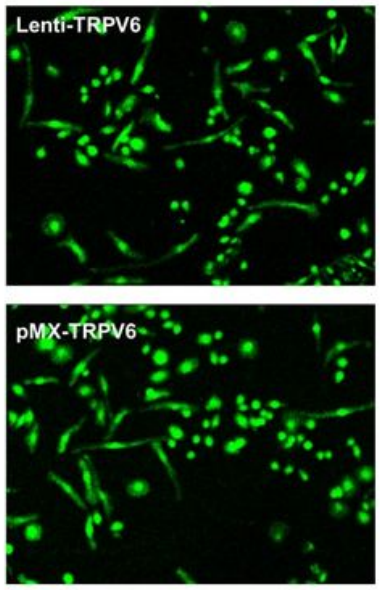

C
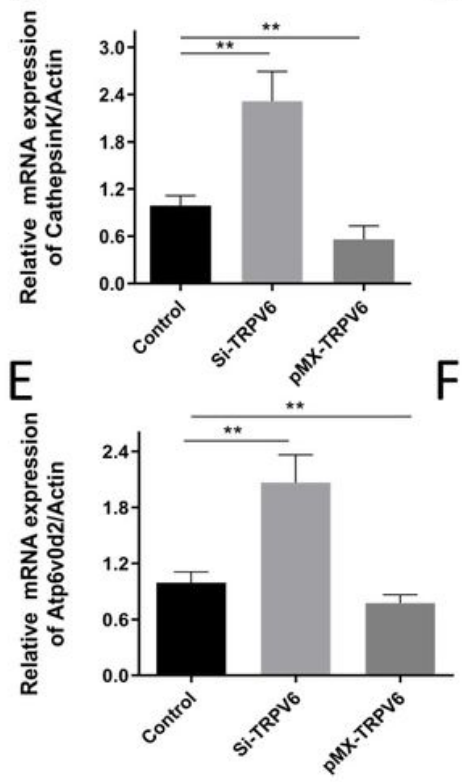

B
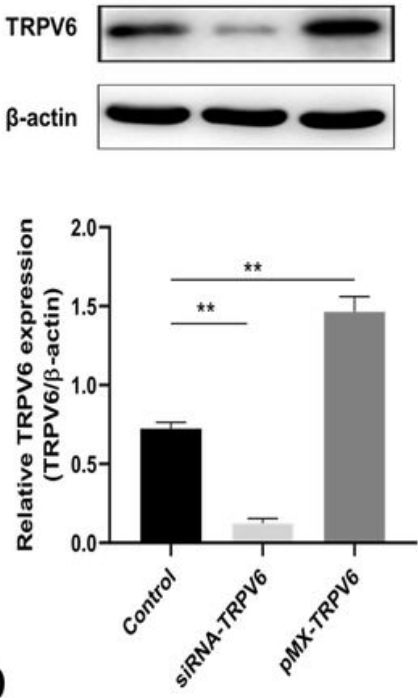

D
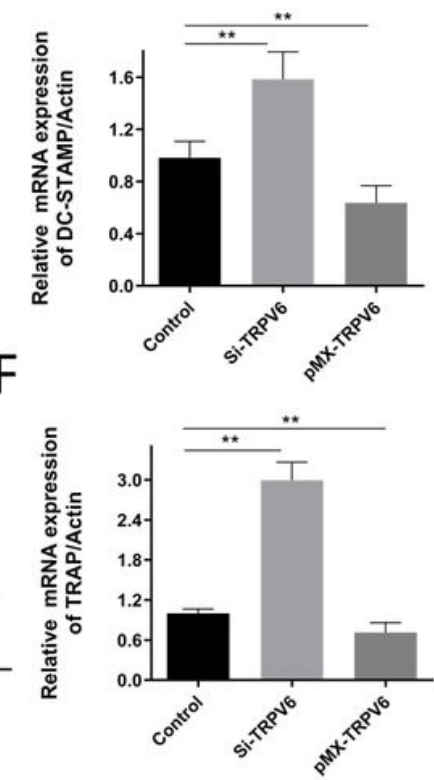

G

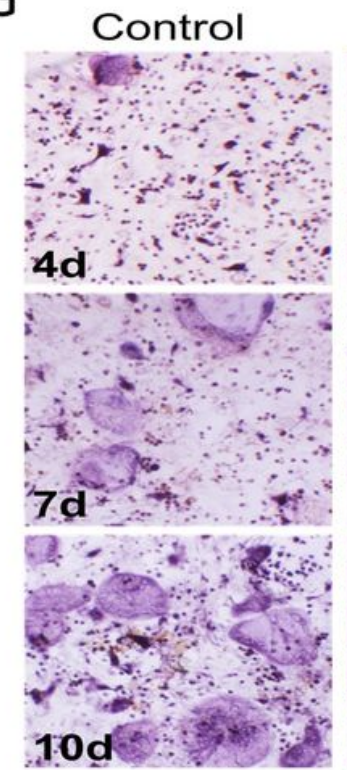

$\mathrm{H}$
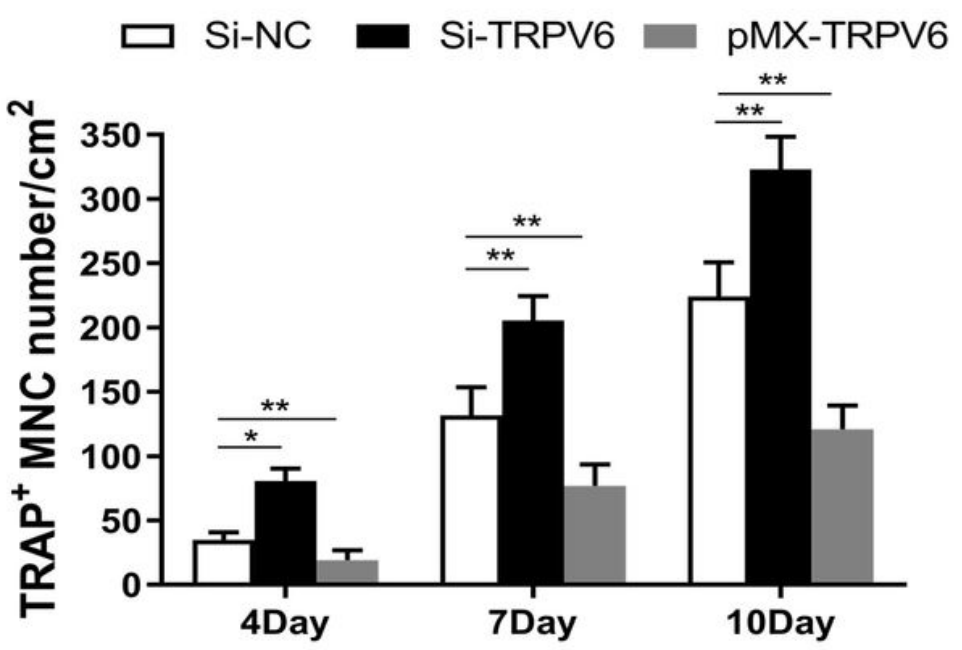

Figure 4

To further verify the role of TRPV6 on osteoclast formation and bone resorption, we silenced and overexpressed TRPV6 gene in osteoclasts by lentivirus transfection. Almost all cells expressed GFP, indicating that the lentivirus transfection rate was more than 95\% (Fig. 4A). Western blotting confirmed that TRPV 6 was effectively silenced and overexpressed (Fig. 4B). The mRNA levels of cathepsin k, DCSTAMP, Atp6v0d2 and TRAP were all increased in osteoclasts infected with lenti-shRNA-TRPV6, whereas were all decreased in the group of osteoclast infected with lenti-pMX-TRPV6 (Fig. 4C-F). Next, 
we assessed the differentiation of osteoclasts with silenced or overexpressed TRPV6 by TRAP staining assay. The number of stained multinuclear TRAP+ osteoclasts was obviously increased in osteoclasts with silenced TRPV6 in a time-dependent manner, whereas osteoclasts showed decreases in the TRPV6overexpressed group (Fig. 4G-H). The above results strongly suggest that TRPV6 was a negative regulator of osteoclast differentiation and function.

A

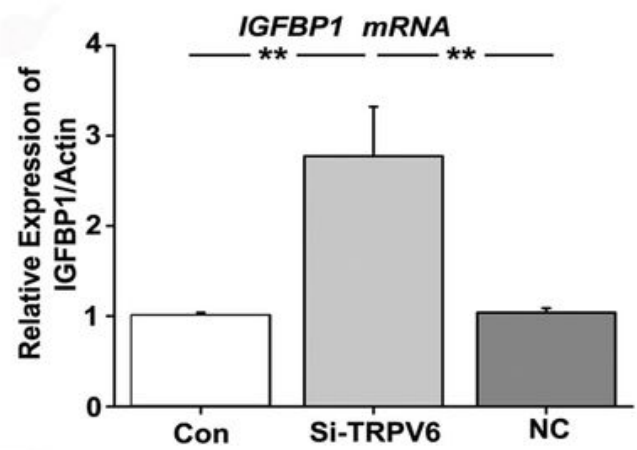

B

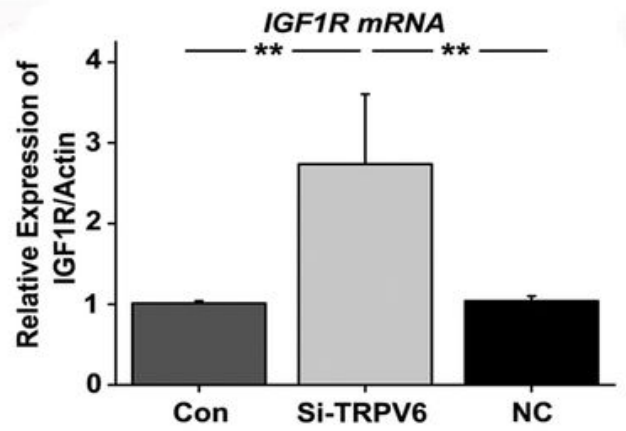

E

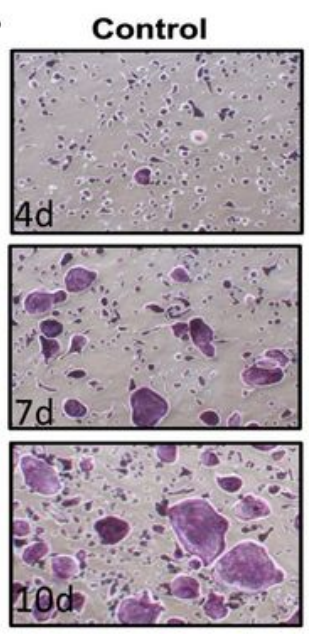

G

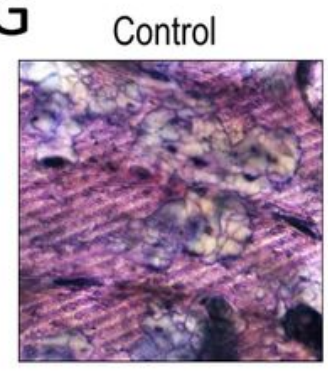

C

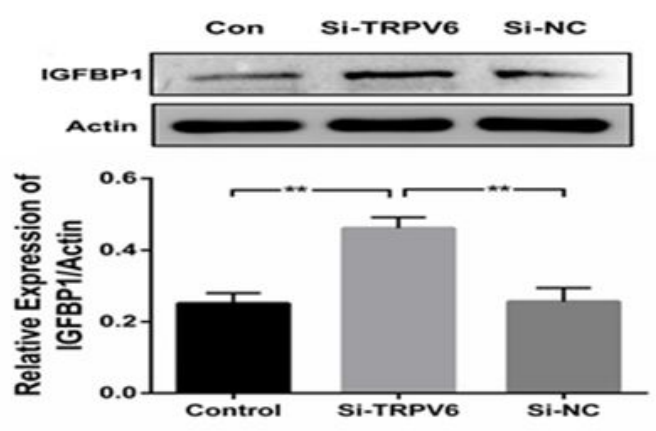

D
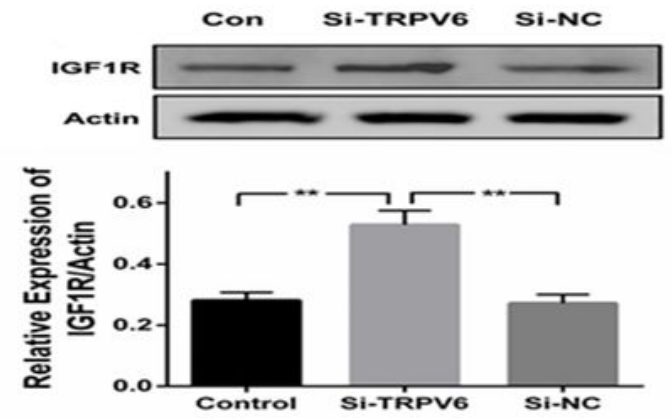

$\mathrm{F}$

Control $\square$ Si-TRPV6 $\square$ Si-TRPV6+NVP

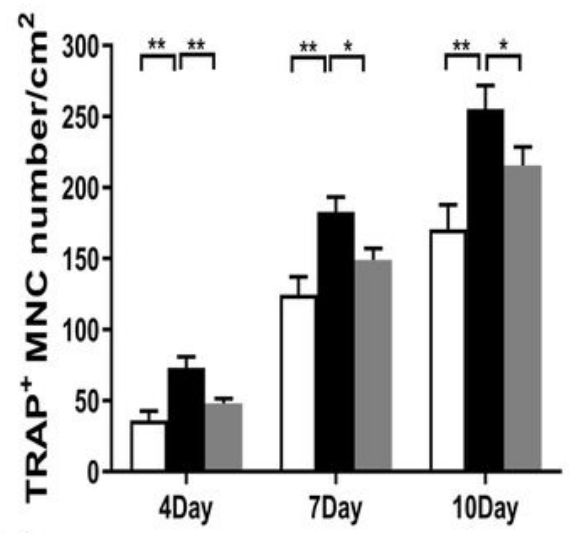

$\mathrm{H}$

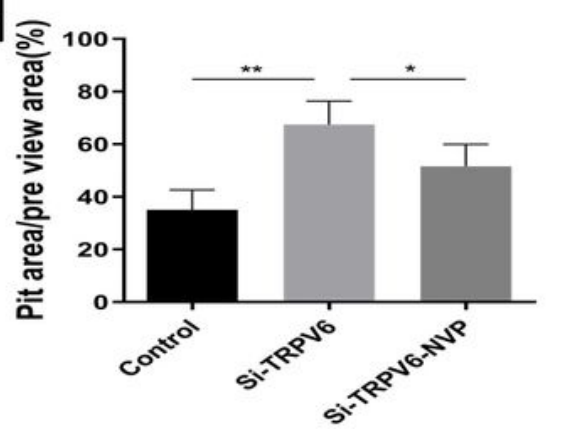

Figure 5 
Our previous study revealed that the RANKL-induced [Ca2+]i oscillation response was not significantly affected by inhibition of TRPV6[16]. As a non-ca2+ oscillating signaling pathway, the IGF pathway plays an important role in osteoclast formation [25-27]. Therefore, we speculated that the IGF signaling pathway was potentially involved in the regulation of osteoclast differentiation and bone absorption by TRPV6. The results showed that the levels of IGF1R and IGFBP1 mRNA and protein in osteoclasts were significantly increased after TRPV6 gene silencing (Fig. 5A-D), which confirmed our speculation. Next, we used an IGF1R antagonist NVP-AEW541 to block the IGF signaling pathway, TRAP staining demonstrated that the inhibitory effect of TRPV6 on osteoclast differentiation was weakened in osteoclasts with blockers (Fig. 5E-F). In accordance with the results for TRAP staining, the IGF1R blocker significantly inhibited the induction of the bone resorption of osteoclasts by silencing of TRPV6, as evidenced by the pit formation assay (Fig. $5 \mathrm{G}-\mathrm{H}$ ). These results strongly suggest that TRPV 6 negatively regulated osteoclast formation and bone resorption by inhibiting the IGF pathway. 
A
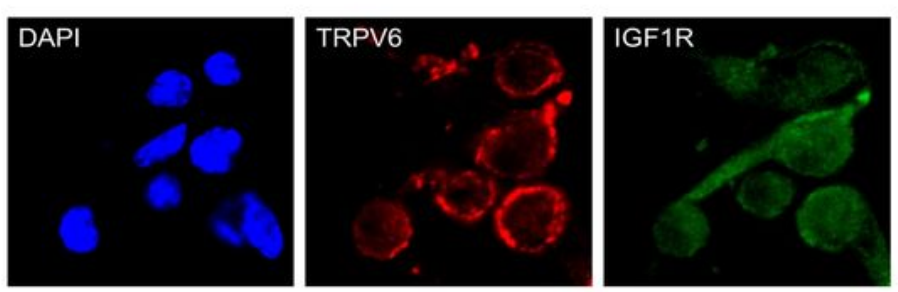

B
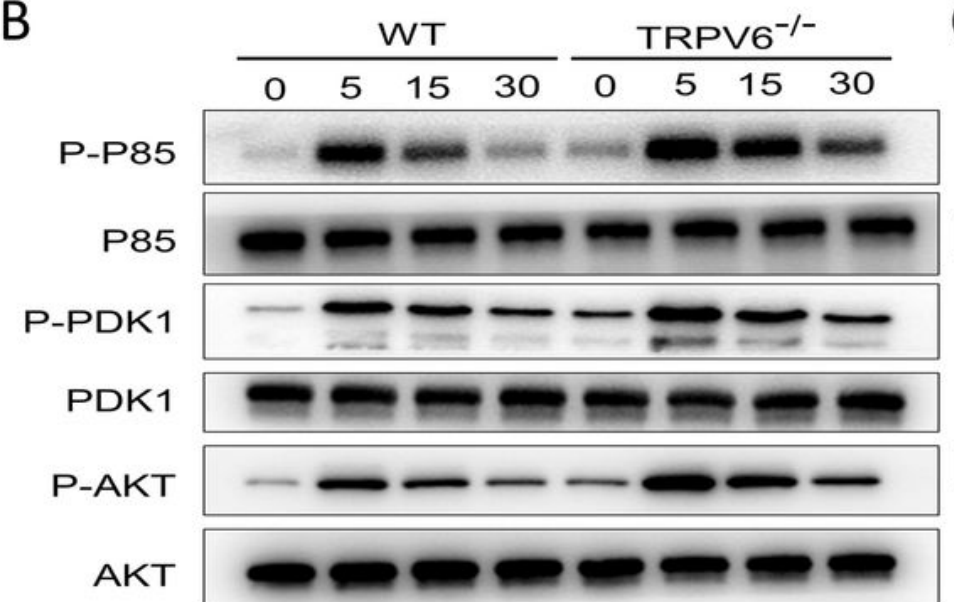

$\beta$-actin

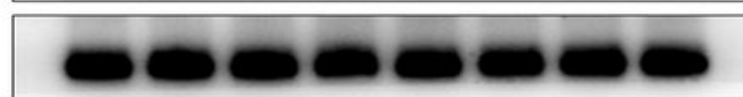

$\mathrm{F}$
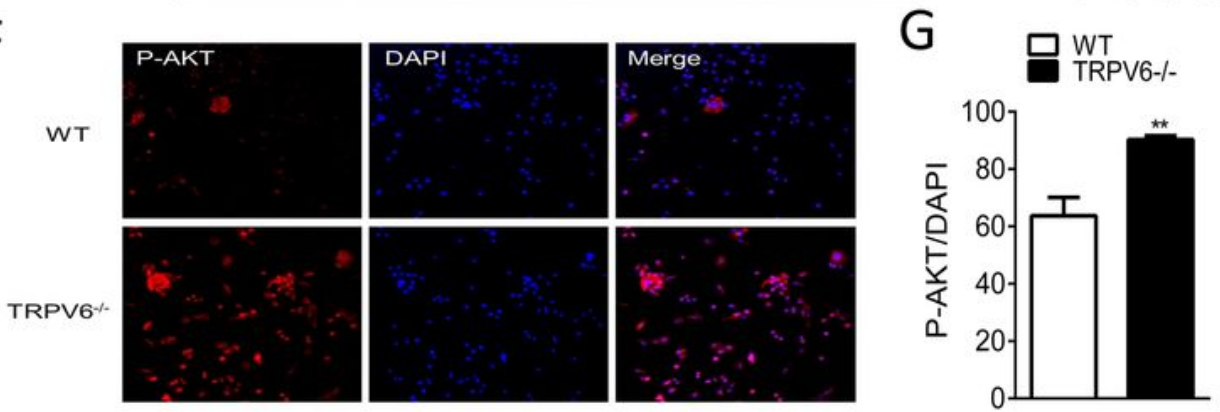

$\mathrm{H}$

\begin{tabular}{|c|c|c|c|}
\hline \multicolumn{2}{|c|}{ Vehicle } & \multicolumn{2}{|c|}{ NVP-AEW541 } \\
\hline WT & TRPV6 $6^{-1-}$ & WT & טחתד \\
\hline
\end{tabular}

P-AKT - - -

AKT

ーーーーーーーーーーーー

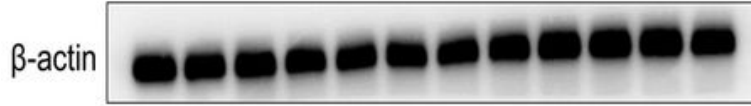

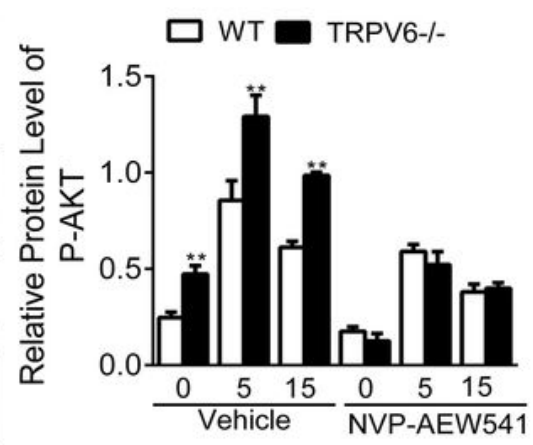

Figure 6

As assessed by immunofluorescence, we found that osteoclasts highly expressed TRPV6 and IGF1R in the membrane and cytoplasm [Fig. 6A]. To further explore the downstream signaling of the IGF pathway, BMMs isolated from TRPV6-/- and WT mice were induced by RANKL and M-CSF for 7 days to form mature osteoclasts. The levels of phosphorylated markers P85/p-P85, PDK1/p-PDK1 and AKT/p-AKT in the PI3K-AKT pathway were detected by western blotting. The results showed that the ratios of P85/p- 
P85, PDK1/p-PDK1 and AKT/p-AKT were increased in osteoclasts isolated from TRPV6-/- mice compared with WT mice [Fig. 6B-E]. Consistent with the western blotting results, immunofluorescence showed that the ratio of PDK1/p-PDK1 of osteoclasts isolated from TRPV6-/- mice was higher than that of osteoclasts isolated from WT mice [Fig. 6F-G]. Next, we used NVP-AEW541 to block the IGF signaling pathway and western blotting revealed that there was no significant difference in the level of AKT/p-AKT between the osteoclasts derived from TRPV6-/- mice and WT mice [Fig. $6 \mathrm{H}-1]$.

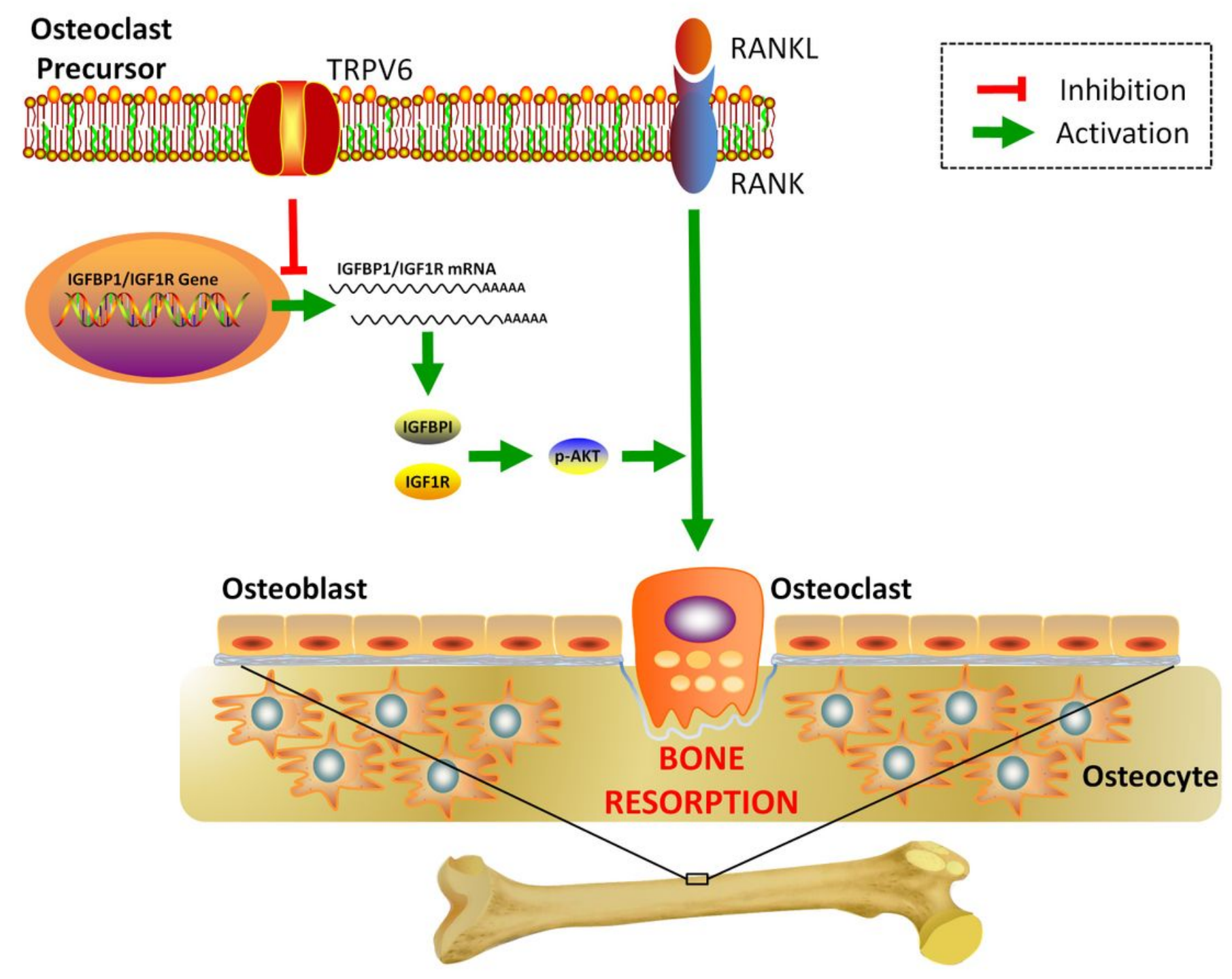

\section{Figure 7}

In the present study, we demonstrated that TRPV6 was a critical negative regulator in RANKL-induced osteoclast differentiation and activity. Our studies showed that TRPV6 decreased osteoclast formation and bone resorption by inhibiting the IGF-PI3K-AKT signaling pathway (Fig. 7). 